\title{
Annual acknowledgement of manuscript reviewers
}

Mario Roberto Dal Poz

\section{Contributing reviewers}

The editors of Human Resources for Health would like to thank all our reviewers who have contributed to the journal in Volume 13 (2015).

\author{
Matilda Aberese-Ako \\ Ghana
}

Mauro Henrique Abreu

Brazil

Elizabeth Adams

Ireland

Orvill Adams

Canada

Adam Ahmat

Congo

Ducksun Ahn

Korea, Republic Of

Mohamad Alameddine

Lebanon

Hasanat Alamgir

USA

Jackeline Alger

Honduras

\section{Robert Alhassan}

Ghana

Fernanda Almeida

Brazil

\section{Alvaro Alonso-Garbayo}

UK

Alla Alsharif

Australia

\author{
Alaa Alsharif \\ Australia
}

Ghanim Y M Alsheikh

UK

Woldekidan Kifle Amde

South Africa

Claire Anderson

Truong Anh Thu

Viet Nam

Laura Antonietti

Argentina

Edson Araujo

USA

Ricardo Arcencio

Brazil

Angel Asúnsolo

Spain

Elsheikh Badr

Sudan

Jane Ball

UK

\section{Allan Barbosa \\ Brazil}

Emma Barnes

UK

\author{
Maria Soledad Barria \\ Chile
}

Robert Basaza

Uganda

Ernesto Báscolo

Argentina

Ken Bassett

Canada

Kisalaya Basu

Canada

N Batura

UK

Lorne Bellan

Canada

\section{Sara Bennett}

USA

David Benton

Switzerland

Miteshkumar Bhanderi India

Kavita Bhatia

India

Aarushi Bhatnagar

India

Posy Bidwell

UK 
Said Bodur

Turkey

Marc Bonenberger

Switzerland

Noemi Bordoni

Argentina

Ivy Bourgeault

Canada

Susan Bradley

UK

Ruairi Brugha

Ireland

James Buchan

Australia

Yvonne Buys

Canada

Johann Cailhol

South Africa

Jennifer Callaghan-Koru

USA

Brian Cameron

Canada

Maureen Canavan

USA

Dora Cardaci

Mexico

Thomas Chacko

India

Yasmin Chandani

Kenya

Yotamu Chirwa

Zimbabwe

Cari Clark

USA Minor Outlying Islands

Helen Cleak

Australia

Rhonda Cockerill

Canada

Sandra Cole

USA

Giorgio Cometto

Switzerland

John Connell

Australia
Sandro Contini

Italy

Jonathan Cowpe

UK

Elaine Daily

USA

Anita Alero Davies

Switzerland

Kenneth De Camargo

Brazil

Willemijn De Graaf-Ruizendaal

Netherlands

Francis Deng

USA

Shrey Desai

India

Ibadat Dhillon

USA

Roland Dimaya

USA

Yan Ding

Germany

Beata Dobrowolska

Poland

Carmen Dolea

Switzerland

Delanyo Dovlo

Namibia

Norbert Dreesch

Austria

Carl-Ardy Dubois

Canada

Christine Duffield

Australia

Gilles Dussault

Portugal

Tom Dyer

UK

Michelle Dynes

USA

Shah Ebrahim

UK

Matt Edwards

UK
Leonie Ellis

Australia

Isabel Emmerick

USA

Boniface Ikenna Eze

Nigeria

Jane Ferguson

UK

Paulo Ferrinho

Portugal

Linda Fogarty

USA

Luiz Eduardo Fonseca

Brazil

Luciana De Barros Correia Fontes Brazil

Alfredo Fort

USA

Sandra Fortes

Brazil

Allison Foster

USA

Allison Annette Foster

USA

Silvia Franzetti

Argentina

Seble Frehywot

USA

Ines Fronteira

Portugal

Diana Frymus

USA

Suzanne Fustukian

UK

Marie-Pierre Gagnon

Canada

Caroline Gaither

USA

Jenny Gallagher

UK

Ana Gama

Portugal

Juan García-Ubaque

Colombia 
Gulin Gedik

Switzerland

Laurent Gerbaud

France

Beatriz Gl Valcarcel

Spain

Irene Glinos

Belgium

Helen Goodyear

UK

Susan Gordon
Australia
William Greene
USA

Gustavo Gusso

Brazil

Bui Ha

Viet Nam

Rohini J Haar

USA

Amy Hagopian

USA

Mythri Halappa

India

Paula Hancock

UK

Christine Hancock

USA

Mark Hann

UK

Piya Hanvoravongchai

Thailand

Janet Harris

UK

Ivonete Heidemann

Brazil

Julie Henderson

Australia

Alison Hernández

Sweden

Peter Heywood

Australia

Peter Hill

Australia
David Hipgrave

Australia

Silvia Hirsch

Argentina

Miriam Hirschfeld

Israel

Stephen Hodgins

USA

Steven Justin Hoffman

Canada

Vincent Homburg

Netherlands

Chee Peng Hor

Malaysia

Kun Huang

China

Sara Hughes

Singapore

Niamh Humphries

Ireland

Anna-Karin Hurtig

Sweden

Kazuo Inoue

Japan

Jehu Iputo

South Africa

Masato Izutsu

Japan

Verughese Jacob

USA

Christel Jansen

Netherlands

Edgar Jarillo

Mexico

Wanda Jaskiewicz

USA

Katrine Jensen

Denmark

Yaping Jin

Canada

Benjamin Johns

USA

Jennifer Johnson

USA
Patrick Kadama

Uganda

Amer Kaissi

USA

László Kalabay

Hungary

Simon Kamau

Kenya

Francis Williams Kamwendo

Malawi

Sumit Kane

Netherlands

Srinivasan Kannan

India

Rebecca Katz

USA

Sheila Keane

Australia

Sosena Kebede

USA

Sunil Khanna

USA

Yohannes Kinfu

Australia

Maxwell Kligerman

USA

Maryse Kok

Netherlands

Adam Koon

UK

Lisbeth Kristiansen

Sweden

Marieke Kroezen

Belgium

Judith Kulig

Canada

Saravana Kumar

Australia

Ramesh Kumar

Pakistan

Rafael Kurtzbart

Argentina

Talma Kushnir

Israel 
Gaetan Lafortune

France

Mylene Lagarde

UK

Michel Landry

USA

Luís Lapão

Portugal

Sarah Larkins

Australia

Elysia Larson

USA

Carole-Lynne Le Navenec

Canada

Yeong Yeh Lee

Malaysia

David Legge

Australia

Uta Lehmann

South Africa

Lia Levin

Israel

Miguelhete Lisboa

Mozambique

Jenny Liu

USA

Alba Llop-Gironés

Spain

Elsbet Lodenstein

Netherlands

Sérgio Lopes

Portugal

Vera Lucia Luiza

Brazil

John Luque

USA

Cristiani Machado

Brazil

Adrian Mackenzie

Canada

Timothy Mackey

USA

Diana Madden

Australia
Elizabeth Madigan

USA

Carinne Magnago

Brazil

Ozayr Mahomed

South Africa

Claudia Maier

USA

Dilip Singh Mairembam

India

Ana Maria Malik

USA

Mats Malqvist

Sweden

Ogenna Manafa

Ireland

Kate Mandeville

UK

Ghulam Farooq Mansoor

Afghanistan

Ludmila Marcinowicz

Poland

Kanchan Marcus

Australia

Susana Margulies

Argentina

Tim Martineau

UK

Grant Martsolf

USA

Keith Masnick

Australia

Leah Masselink

USA

Andrea Veronica Mastrangelo

Argentina

Maria Mathews

Canada

Verona Mathews

South Africa

Josue Mbonigaba

South Africa

Willy Mccourt

UK
Matthew Mcgrail

Australia

Pamela Mcquide

Namibia

Joanne Mcveigh

Ireland

Araya Abrha Medhanyie

Ethiopia

Hugo Mercer

Argentina

Edward Mills

Canada

Mario Monteiro

Brazil

Anthony Montgomery

Greece

Jean Moore

USA

Joanna Morrison

UK

Frederick Mugisha

Uganda

Amin Muhammad Gadit

Canada

Wilbroad Mutale

Zambia

Janet Myers

USA

Lucio Naccarella

Australia

Takashi Nagata

Japan

Papreen Nahar

UK

Joel Negin

Australia

Anne Nesbitt

Sierra Leone

Jonathan Newbury

Australia

Ha Nguyen

USA

Gustavo Nigenda

Mexico 
Mimi Niles

USA

Mwansa Annette Nkowane

Switzerland

\section{Godswill Nnaji}

Nigeria

Charles Normand

Ireland

Mary Patricia Nowalk

USA

Humphreys Nsona

Malawi

Jennifer Nyoni

Congo

Jerry Okal

Kenya

Larry Olsen

USA

Pauline Oosterhoff

Netherlands

Carole Orchard

Canada

Francisco Ortega

Brazil

Belinda O'sullivan

Australia

Susanne Ozegowski

Germany

Nermin Ozgulbas

Turkey

Ligia Paina

Elisabeth Paul

Belgium

Mark Pauly

USA

Matthew Pearce

USA

Ann Pederson

Canada

Marina Peduzzi

Brazil

\section{Senga Pemba}

Tanzania
Ha Pham

Viet Nam

Pudtan Phanthunane

Thailand

Anastas Philalithis

Greece

Yogan Pillay

South Africa

Victor Piriz

Uruguay

Patricia Pittman

USA

Evgeniya Plotnikova

UK

Kaja Polluste

Estonia

María Pozzio

Argentina

Ari Probandari

Indonesia

Eduardo Benjamin Puertas

Ecuador

Mary Purkis

Canada

Weerasak Putthasri

Thailand

Lal Rawal

Bangladesh

Stephen Reid

South Africa

Michelle Remme

UK

Thomas Ricketts

USA

Jemimah Ride

Australia

Monika Riedel

Austria

Patricia Riley

USA

Laetitia Rispel

South Africa

Bjarne Robberstad

Norway
Priscilla Robinson

Australia

Jose L. Rocha

Spain

Wesley Rohrer

Saudi Arabia

Janetta Roos

South Africa

Ellen Roskam

Switzerland

Arie Rotem

Australia

Giuliano Russo

Portugal

Gayle Rutherford

Canada

Dana Ryan

Canada

Mandy Ryan

UK

Bukola Salami

Canada

Fabiano Saldanha Gomes De Oliveira Brazil

Domenico Salvatore

Italy

Delia Sanchez

Uruguay

Teresa Sanchez-Sagrado

France

Milena Santric Milicevic

Serbia

Eric Sarriot

USA

María Cecilia Scaglia

Argentina

Marta Schaaf

USA

Richard Scheffler

USA

Andreas Schmid

Germany

Philip Seidenberg

USA 
Tarun Sen Gupta

Australia

Walter Sermeus

Belgium

P Ravi Shankar

Nepal

Amani Shao

Tanzania

John Shaw

New Zealand

Zubin Shroff

Switzerland

Jonathan Sicsic

France

Mohsin Sidat

Mozambique

Martin Silberman

Argentina

Daudi Simba

Tanzania

Donald Simpson

USA

Amani Siyam

Switzerland

Marion Slack

USA

Martin Smith

South Africa

Jeffrey Soar

Australia

Julie Sochalski

USA

Nils Gunnar Songstad

Norway

Michael Spencer

USA

Allison Squires

USA

Barbara Stilwell

USA
Sabine Stordeur

Belgium

Roger Strasser

Canada

Tara Tancred

UK

Shenglan Tang

USA

Akhenaten Tankwanchi

USA

Katherine Taylor

UK

Conor Teljeur

Ireland

Petra Ten Hoope-Bender

Switzerland

Kea Tijdens

Netherlands

Vandana Tripathi

USA

Eralda Turkeshi

Albania

Liina-Kaisa Tynkkynen

Finland

Graciela Uriburu

Argentina

Peter Van Bogaert

Belgium

Hendrik P. Van Dalen

Netherlands

Anke Van Der Kwaak

Netherlands

Paula Vassallo

Malta

Vanesa Vazquez Laba

Argentina

Marko Vujicic

USA

Qun Wang

China
Rachel J Waxman

USA

William Weeks

USA

Madeleen Wegelin

Netherlands

William Willis

USA

Kim Wilson

USA

Nancy Winslade

Canada

Christiane Wiskow

Switzerland

Matthias Wismar

Belgium

Kelley Withy

USA

Timothy Wood

Australia

Scott Wright

USA

Ansgar Wuebker

Germany

Kaspar Wyss

Switzerland

Mezgebu Yitayal

Ethiopia

Beibei Yuan

Sweden

Junhua Zhang

China

Rose Zulliger

USA

Joseph Zulu

Zambia

Prisca Ac Zwanikken

Netherlands 